\title{
Effect of improving representation of horizontal and vertical cloud structure on the Earth's global radiation budget. Part I: review and parametrization
}

Article

Published Version

Shonk, J. K. P., Hogan, R. J., Edwards, J. M. and Mace, G. G. (2010) Effect of improving representation of horizontal and vertical cloud structure on the Earth's global radiation budget. Part I: review and parametrization. Quarterly Journal of the Royal Meteorological Society, 136 (650). pp. 1191-1204. ISSN 1477-870X doi: https://doi.org/10.1002/qj.647 Available at https://centaur.reading.ac.uk/16261/

It is advisable to refer to the publisher's version if you intend to cite from the work. See Guidance on citing.

To link to this article DOI: http://dx.doi.org/10.1002/qj.647

Publisher: Royal Meteorological Society

All outputs in CentAUR are protected by Intellectual Property Rights law, including copyright law. Copyright and IPR is retained by the creators or other copyright holders. Terms and conditions for use of this material are defined in the End User Agreement. 


\section{www.reading.ac.uk/centaur}

\section{CentAUR}

Central Archive at the University of Reading

Reading's research outputs online 


\title{
RMetS
}

Royal Meteorological Society

\section{Effect of improving representation of horizontal and vertical cloud structure on the Earth's global radiation budget. Part I: Review and parametrization}

\author{
Jonathan K. P. Shonk, ${ }^{a \star}$ Robin J. Hogan, ${ }^{a}$ John M. Edwards ${ }^{b \dagger}$ and Gerald G. Mace ${ }^{c}$ \\ ${ }^{a}$ Department of Meteorology, University of Reading, $U K$ \\ ${ }^{\mathrm{b}}$ Met Office, Exeter, UK \\ ${ }^{c}$ Department of Meteorology, University of Utah, Salt Lake City, USA \\ ${ }^{\star}$ Correspondence to: Jonathan Shonk, Department of Meteorology, University of Reading, Earley Gate, Reading RG6 \\ 6BB, Berkshire, UK. E-mail: j.k.p.shonk@reading.ac.uk \\ ${ }^{\dagger}$ The contribution of J. M. Edwards was written in the course of his employment at the Met Office, UK, and is published \\ with the permission of the Controller of HMSO and the Queen's Printer for Scotland.
}

A poor representation of cloud structure in a general circulation model (GCM) is widely recognised as a potential source of error in the radiation budget. Here, we develop a new way of representing both horizontal and vertical cloud structure in a radiation scheme. This combines the 'Tripleclouds' parametrization, which introduces inhomogeneity by using two cloudy regions in each layer as opposed to one, each with different water content values, with 'exponential-random' overlap, in which clouds in adjacent layers are not overlapped maximally, but according to a vertical decorrelation scale. This paper, Part I of two, aims to parametrize the two effects such that they can be used in a GCM. To achieve this, we first review a number of studies for a globally applicable value of fractional standard deviation of water content for use in Tripleclouds. We obtain a value of $0.75 \pm 0.18$ from a variety of different types of observations, with no apparent dependence on cloud type or gridbox size. Then, through a second short review, we create a parametrization of decorrelation scale for use in exponential-random overlap, which varies the scale linearly with latitude from $2.9 \mathrm{~km}$ at the Equator to $0.4 \mathrm{~km}$ at the poles. When applied to radar data, both components are found to have radiative impacts capable of offsetting biases caused by cloud misrepresentation. Part II of this paper implements Tripleclouds and exponential-random overlap into a radiation code and examines both their individual and combined impacts on the global radiation budget using re-analysis data. Copyright (c) 2010 Royal Meteorological Society and Crown Copyright.

Key Words: cloud inhomogeneity; overlap; decorrelation height; Tripleclouds; exponential-random.

Received 13 July 2009; Revised 31 March 2010; Accepted 12 April 2010; Published online in Wiley InterScience 23 July 2010

Citation: Shonk JKP, Hogan RJ, Edwards JM, Mace GG. 2010. Effect of improving representation of horizontal and vertical cloud structure on the Earth's global radiation budget. Part I: Review and parametrization. Q. J. R. Meteorol. Soc. 136: 1191-1204. DOI:10.1002/qj.647

\section{Introduction}

Clouds are a very important part of the Earth's radiation budget, and the interaction of clouds with radiation has significant effects on climate. However, on account of the complex processes that determine a cloud's evolution, and the complicated structure that exists within it, modelling clouds in a reasonable manner remains the greatest challenge in climate modelling (Randall, et al., 2007). Part of this challenge is presented by the sensitivity of the radiation 
budget to cloud properties (e.g. Slingo, 1990). In turn, on account of the inextricable connection between clouds and radiation, there is a large uncertainty in the magnitude and sign of the cloud feedback (Colman, 2003; Webb, et al., 2006). In order to make progress, it is imperative that we represent clouds as realistically as possible in climate models.

Both macroscale structure, such as vertical alignment of clouds in their gridboxes (Wu and Moncrieff, 2001), and microscale structure, such as variation of ice crystal size ( $\mathrm{Gu}$ and Liou, 2006), can lead to significant biases being introduced into the Earth's radiation budget. In terms of representing cloud structure, the issue can be partitioned into two parts: firstly, we need a reliable representation of horizontal cloud structure; and secondly, we require a realistic parametrization describing how the cloud aligns vertically. Barker, et al. (1999) found poor representation of either component to generate large radiative biases when evaluated with a radiation code, with error contributions of different signs from the two components.

Most modern general circulation models (GCMs) represent clouds using the plane-parallel approximation: a single homogeneous region of cloud in each gridbox layer. While the plane-parallel approximation is computationally efficient, removing cloud structure has detrimental effects on the interaction between clouds and radiation, both in the short-wave (e.g. Barker and Davies, 1992; Carlin, et al., 2002) and the long-wave (e.g. Pomroy and Illingworth, 2000), creating positive biases in short-wave albedo and long-wave emissivity of order $10 \%$ on average.

Several solutions to the problem of plane-parallel biases have been proposed. One suggestion was to scale the cloud's optical properties in order that its interaction with radiation creates more realistic fluxes (Davis, et al., 1990; Cahalan, et al., 1994; Cairns, et al., 2000). This method is efficient, but a single value of scaling factor was found to be inappropriate for all cases, as its optimum value is dependent on many factors, including horizontal gridbox size (Pomroy and Illingworth, 2000) and spectral region (Yu, et al., 1997). It was also found to have a range of different values across the world, according to Oreopoulos and Cahalan (2005). Another suggested approach involves statistically replicating the inhomogeneity by weighting the cloud optical depth by a distribution function. Barker (1996) modified the twostream radiative transfer equations at a fundamental level to weight optical depth by a gamma distribution, which was found by Carlin, et al. (2002) to dramatically reduce the plane-parallel biases. A gamma-weighting method proposed by Oreopoulos and Barker (1999) produced similar results, but with increased computational efficiency by applying the weighting to the radiation transfer coefficients.

A simple method to introduce inhomogeneity would be to use a multi-column independent column approximation (ICA) calculation. However, this has the major disadvantage of introducing significant additional computation time. This can be reduced by temporal sampling, although the increase in computation time could still be large. Pincus, et al. (2003) recognised that this time increase was caused by the double integral over space and wavelength, and suggested an ICA-type calculation that simultaneously sampled wavelength and cloud state, therefore creating a calculation that required exactly the same time to perform as a plane-parallel calculation. This method, referred to as the Monte-Carlo Independent Column Approximation (McICA), has been implemented in the forecast system of the European Centre for Medium-Range Weather Forecasts (ECMWF; Morcrette, et al., 2008) and the ECHAM-5 model (Räisänen, et al., 2007).

While the McICA method is computationally efficient, the output fluxes suffer from an amount of random noise. This is caused by the random nature of the allocation of spectral region to cloud state, and the randomness of the stochastic cloud generator used to generate the cloud states (Räisänen, et al., 2004). This noise was only found to have an effect on climate simulations when the level of noise was artificially high (Räisänen, et al., 2007). An alternative method was proposed by Shonk and Hogan (2008). Known as 'Tripleclouds', the cloud scheme represents cloud inhomogeneity by introducing a second cloudy region into each layer of a gridbox. One of the two cloudy regions represents the optically thinner cloud in that layer; the other represents the optically thicker cloud. The Tripleclouds scheme does not suffer the effects of random noise. However, on account of the added complexity of solving radiative transfer through an extra region in each layer, the calculation is found to take about $25 \%$ longer. When applied to radar data, Shonk and Hogan (2008) found the Tripleclouds scheme to reduce an $8 \%$ planeparallel albedo bias to less than $1 \%$, with a much better performance than applying a scaling factor. The scheme was found to have no particular dependence on horizontal gridbox size, vertical resolution or solar zenith angle. It was also found to reduce root-mean-square heating rate errors.

In terms of vertical structure, most current models tend to align these plane-parallel clouds using 'maximum-random' overlap (Geleyn and Hollingsworth, 1979). In this method, vertically continuous cloud is overlapped maximally, and cloud layers separated by at least one layer of clear sky are overlapped randomly. While the performance of maximumrandom overlap was advocated by the study of Tian and Curry (1989), Barker, et al. (1999) found that overlapping vertically continuous clouds this way still caused biases to be introduced. This implies that, for a pair of cloudy layers, the cloud should be aligned such that its cloud cover is somewhere between that of maximum-random and random overlap.

Hogan and Illingworth (2000) analysed cloud radar data from Chilbolton in Hampshire, southern England, to derive cloud overlap statistics. They introduced an 'overlap parameter' $\alpha$, which quantified the degree of correlation between horizontal cloud positions in two cloudy layers and had a value of zero for random overlap and a value of one for maximum overlap. They determined values of $\alpha$ for pairs of cloudy model layers and found that, while random overlap described the overlap between cloud in separated cloud layers, the value of $\alpha$ had an exponential relationship with the separation height that was less than maximum, in agreement with Barker, et al. (1999). Using this 'exponential-random' overlap in a GCM should therefore improve its ability to represent vertical cloud structure.

In this two-part paper, we aim to generate a full parametrization of cloud structure that is suitable for use in a GCM, combining the Tripleclouds horizontal inhomogeneity parametrization of Shonk and Hogan (2008) with the exponential-random vertical overlap parametrization of Hogan and Illingworth (2000). Through a review of a number of studies into both horizontal and vertical cloud structure, this paper (hereafter 'Part I') builds the parametrization and modifies it to make it globally 
applicable. The Tripleclouds method, described in full by Shonk and Hogan (2008), was developed using radar data, and the statistics to describe the two water content values in each layer determined exactly from the predetermined distribution function. We need to modify this method such that the two values can be determined from the single value of water content available in the model data.

Section 2 of this paper contains an in-depth review of cloud inhomogeneity, comparing statistics of variability of in-cloud water content and optical depth derived from a range of data sources, both ground-based and space-based. From this review, a quantitative, globally applicable estimate of this variability is obtained. This is used to determine the pair of water content values in the Tripleclouds scheme and is subsequently verified using radar data. Section 3 then reviews papers on vertical structure using data from groundbased radar and derives a simple equation describing the global distribution of the nature of cloud overlap. This is then applied to exponential-random overlap and also tested on radar data. In the second part of this paper (Shonk and Hogan (2010), hereafter referred to as 'Part II'), we apply Tripleclouds and exponential-random overlap to global model re-analysis data from the ECMWF to evaluate the performance of the two components. We also separately consider the effects on global radiation budget of implementing the horizontal and vertical parametrizations.

\section{Horizontal inhomogeneity parametrization}

The original Tripleclouds method used by Shonk and Hogan (2008) to calculate the pair of water content values in a given gridbox layer is demonstrated in Figure 1(a). The lower water content value is determined by the 16th percentile of the continous distribution in that layer, and the higher value is chosen to conserve the mean. This method (referred to hereafter as the 'lower percentile (LP) method'), however, is only applicable when the water content distribution is known, as was the case for the radar data used. For a GCM, water content is stored only as a single mean value, with no information regarding the shape of the distribution. This implies that an alternative method is required.

The studies discussed in the previous section all attempted to quantify cloud inhomogeneity using a range of different sets of observational data from a range of different platforms (satellite, radar and aircraft), for various locations and cloud types. In this section, results and statistics from some of these studies are compared by converting their various measures of cloud variability into a single statistical quantity. We use fractional standard deviation, $f$. For a variable $x$, fractional standard deviation is defined as the standard deviation of $x$ divided by its mean value:

$$
f_{x}=\frac{\sigma_{x}}{\bar{x}}
$$

In section 2.1, previous measures of cloud variability are converted to values of fractional standard deviation of either layer-by-layer water content $\left(f_{w}\right)$, or vertically integrated optical depth $\left(f_{\tau}\right)$ or water path $\left(f_{w}\right)$. These studies are then compared in section 2.2, with the aim of generating a simple measure of horizontal cloud variability that is globally applicable and can be used in the Tripleclouds parametrization. It will allow a pair of water content values, $w_{\text {TC }}$, to be defined from a single mean water content value
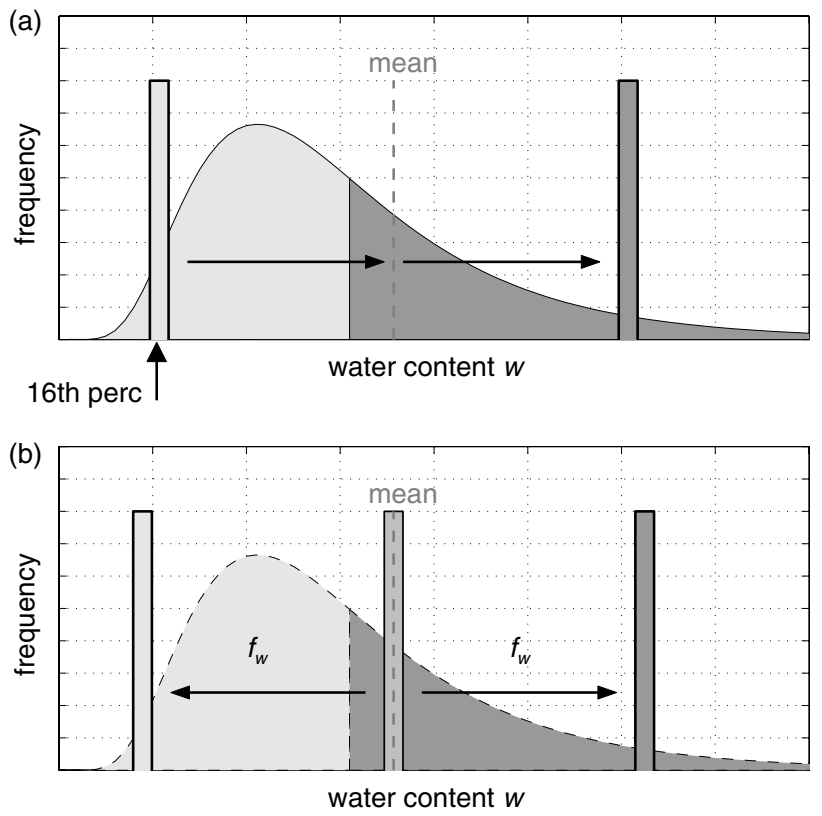

Figure 1. Schematic showing the calculation of the two water content values required for Tripleclouds using (a) the original lower percentile (LP) method of Shonk and Hogan (2008) and (b) the fractional standard deviation (FSD) method used in this study. The shaded area represents the arbitrary distribution of water content in a gridbox layer. In both methods, this distribution is partitioned into two regions through the median, with the two bars (pale grey and dark grey) representing the cloud water content in each region. For the LP method, the lower water content value is determined by the 16th percentile of the distribution, and the higher value is chosen to conserve the mean. In the FSD method, the exact shape of the distribution (shown dashed) is unknown; the available information consists only of the mean value, shown by the single bar at the mean water content. The pair of values are determined by adding and subtracting one fractional standard deviation to this mean value, shown by the pale grey and dark grey bars in (b). For mixed-phase clouds, this method is applied separately to ice and liquid water content.

$\bar{w}$ via the equation:

$$
w_{\mathrm{TC}}=\bar{w} \pm f_{w} \bar{w}
$$

This method, referred to as the 'fractional standard deviation (FSD) method', is shown diagrammatically in Figure 1(b). The applicability of (2) is subsequently tested in a radiation scheme in section 2.3 .

\subsection{Previous measures of cloud inhomogeneity}

The scaling factor $\chi$ of Cahalan, et al. (1994) (and others) is related to both the mean and spread of the values of optical depth in a gridbox, according to their Eq. (3.9), which gives the definition of the scaling factor as:

$$
\chi=\frac{\exp \{\overline{\ln (\tau)}\}}{\bar{\tau}}
$$

assuming that the effective radius of the water droplets is much less variable than optical depth. The derivation of fractional standard deviation from this scaling factor can be performed simply by expressing the statistical properties of optical depth in terms of a two-point PDF, such as that used in Tripleclouds, with the two optical depth values separated by one standard deviation. For a discrete two-point PDF of $\tau$ with two values of optical depth, $\bar{\tau}+\sigma_{\tau}$ and $\bar{\tau}-\sigma_{\tau}$, (3) 
can be written as:

$$
\chi \bar{\tau}=\exp \left[\frac{\ln \left(\bar{\tau}-\sigma_{\tau}\right)+\ln \left(\bar{\tau}+\sigma_{\tau}\right)}{2}\right] .
$$

With further manipulation, this becomes:

$$
f_{\tau}=\frac{\sigma_{\tau}}{\bar{\tau}}=\sqrt{1-\chi^{2}} .
$$

Using this equation, we can directly convert the values of scaling factor estimated from observations by Cahalan, et al. (1994) and Oreopoulos and Cahalan (2005) to values of $f_{\tau}$.

The definition of renormalisation parameter $\epsilon$ used by Rossow, et al. (2002), based on the work of Cairns, et al. (2000), does not allow straightforward conversion to fractional standard deviation. Rossow, et al. (2002) describe the relationship between renormalisation parameter and scaling factor as:

$$
\epsilon=1-\chi_{0}
$$

However, the scaling factor $\chi_{0}$ is defined differently to the factor $\chi$ that was used by Cahalan, et al. (1994). This difference is explained by Rossow, et al. (2002), and comparisons are drawn between the two by Oreopulos and Davies (1998). Multiplying the linear mean optical depth $\bar{\tau}$ by the optimum value of scaling factor $\chi_{0}$ should give an effective mean value that is equal to the 'radiative mean' optical depth; that is, the value of optical depth that gives the true mean spherical albedo or emissivity. Performing the same process with an optimum value of scaling factor $\chi$ will produce an optical depth that is equal to the 'logarithmic mean' optical depth, as shown in (3). This will give correct radiative fluxes when using a log-normal distribution of optical depth, but may not give exact results when applied to real data. While these two definitions differ slightly, it is often fair to approximate distributions of water content to be log-normal (Hogan and Illingworth, 2003) and hence assume that $\chi \approx \chi_{0}$. In other words, we can say:

$$
\epsilon \approx 1-\chi
$$

with the condition that there is an element of uncertainty. Conversion from $\chi$ to $f_{\tau}$ can then be performed using (5).

Barker, et al. (1996) described the cloud variability in their study in terms of the gamma distribution variance parameter $v$. This is simply calculated from the mean and standard deviation of optical depth across each scene. Conversion from $v$ to $f_{\tau}$ is therefore equally straightforward:

$$
f_{\tau}=v^{-1 / 2} \text {. }
$$

Pincus, et al. (1999) defined their cloud variability as 'dispersion', which is equivalent to fractional standard deviation of the $\log$ of optical depth, $f_{\ln (\tau)}$. They also report the mean of the $\log$ of optical depth, $\overline{\ln (\tau)}$. From these quantities, we can extract the fractional standard deviation of optical depth using:

$$
f_{\tau}=f_{\ln (\tau)} \overline{\ln (\tau)} .
$$

Our comparisons also include the cloud variability measured by Smith and DelGenio (2001). Their linear relationship between mean ice water content and its standard deviation led to a simple derivation of $f_{w}$ by evaluating the gradient of the best fit line. We also include calculations of $f_{w}$ for overcast ice clouds using the empirical formula of Hogan and Illingworth (2003):

$$
\log _{10}\left(f_{\mathrm{w}}{ }^{2}\right)=0.3 \log _{10}(\Delta x)-0.04 s-0.93,
$$

where $\Delta x$ is the gridbox size in kilometres and $s$ is the vertical wind shear in $\mathrm{m} \mathrm{s}^{-1} \mathrm{~km}^{-1}$. This equation applies for $\Delta x<60 \mathrm{~km}$; for any larger gridbox sizes, $\Delta x$ is fixed to $60 \mathrm{~km}$. Our calculated values use a fixed vertical shear $s$ of $5 \mathrm{~m} \mathrm{~s}^{-1} \mathrm{~km}^{-1}$ and gridbox sizes $\Delta x$ of $50 \mathrm{~km}, 100 \mathrm{~km}$ and $200 \mathrm{~km}$. The statistics derived from the data used by Shonk and Hogan (2008) are also included in this comparison.

\subsection{Comparison of the observations}

The values of fractional standard deviation $f$ are compared in Table I. All values have been derived from cloudy pixels alone; no clear-sky pixels have been included in the calculations. On account of the wide variety of studies compared here, we separate the values of $f$ into a number of different categories, given at the top of the table. These categories are now explained.

- Source. The platform from which the data were collected to derive the value of $f$. In the studies compared here, the values are derived from radar data, aircraft measurements, microwave radiometer measurements and satellite observations.

- Location. The latitude range in which the observations were made.

- Season. Whether the value applies to the winter hemisphere, summer hemisphere or a combination.

- Surface. Whether the value applies to cloud over land or cloud over the ocean.

- Cloud type. Description of the type of cloud to which the value applies.

- Variable. The measured cloud quantity that the value is derived from: either ice or liquid water content $\left(w_{\mathrm{i}}\right.$ or $\left.w_{1}\right)$; liquid water path $\left(W_{1}\right)$; or optical depth $(\tau)$.

Also, the gridbox sizes that the raw data were divided into for analysis, and the pixel size used to investigate the variability, are included. For the studies with radar data, this gridbox size was converted approximately from a temporal size to a physical size by assuming a wind speed typical to the level of the clouds in question ( $10 \mathrm{~m} \mathrm{~s}^{-1}$ for liquid clouds; $15 \mathrm{~m} \mathrm{~s}^{-1}$ for ice clouds).

Despite the diverse nature of the data shown, the values of fractional standard deviation are relatively similar, with most of the values in the range 0.5 to 0.9 . By inspection, it is found that there is little evidence of trends in variability associated with the different categories. For example, there is no apparent relationship between $f$ and season or surface type, although inspection of the values observed by Oreopoulos and Cahalan (2005) shows that, over the ocean, winter clouds tend to have higher values of $f$, while over the land, the summer clouds tend to have higher values. This is caused by a combination of effects from tropical convection over land and cloud in midlatitude weather systems.

Figure 2 plots the fractional standard deviation values as a function of the data gridbox size. Where there was more than one gridbox size evaluated, this range is shown by the vertical error bars; for the radar studies, the vertical error 
Table I. List of mean fractional standard deviations $f$, derived from a number of studies using different data sources. The categories used in the column headings are described in the text.

\begin{tabular}{|c|c|c|c|c|c|c|c|c|}
\hline Reference; source & Location & Season & Surface & Cloud type & Variable & $\begin{array}{l}\text { Gridbox } \\
\text { size }(\mathrm{km})\end{array}$ & $\begin{array}{c}\text { Pixel } \\
\text { size }(\mathrm{km})\end{array}$ & $\begin{array}{l}\text { Mean } \\
\quad f\end{array}$ \\
\hline \multirow{2}{*}{$\begin{array}{l}\text { Shonk and Hogan } \\
(2008) ; \text { radar/MWR }\end{array}$} & midlatitude & both & land & ice & $w_{\mathrm{i}}$ & $\sim 112.5$ & $\sim 2.25$ & 0.965 \\
\hline & midlatitude & both & land & liquid & $w_{1}$ & $\sim 75$ & $\sim 1.5$ & 0.742 \\
\hline \multirow{3}{*}{$\begin{array}{l}\text { Hogan and } \\
\text { Illingworth (2003); } \\
\text { radar }\end{array}$} & midlatitude & both & land & cirrus & $w_{\mathrm{i}}$ & $50^{\star}$ & $\sim 0.45$ & 0.490 \\
\hline & midlatitude & both & land & cirrus & $w_{\mathrm{i}}$ & $100^{*}$ & $\sim 0.45$ & 0.503 \\
\hline & midlatitude & both & land & cirrus & $w_{\mathrm{i}}$ & $200^{\star}$ & $\sim 0.45$ & 0.503 \\
\hline $\begin{array}{l}\text { Cahalan et al. (1994); } \\
\text { MWR }^{\dagger}\end{array}$ & midlatitude & summer & ocean & liquid & $W_{1}$ & $\sim 15000^{\dagger}$ & $\sim 6$ & 0.714 \\
\hline $\begin{array}{l}\text { Smith and DelGenio } \\
\text { (2001); aircraft }\end{array}$ & midlatitude & winter & land & cirrus & $w_{\mathrm{i}}$ & 175 & 0.8 & 0.858 \\
\hline \multirow{3}{*}{$\begin{array}{l}\text { Barker et al. (1996); } \\
\text { satellite (LandSat) }\end{array}$} & midlatitude & summer & ocean & broken $\mathrm{Sc}^{\ddagger}$ & $\tau$ & 58 & 0.042 & 0.994 \\
\hline & midlatitude & summer & ocean & overcast $\mathrm{Sc}^{\ddagger}$ & $\tau$ & 58 & 0.042 & 0.445 \\
\hline & midlatitude & both & ocean & cumulus & $\tau$ & 58 & 0.042 & 1.374 \\
\hline \multirow{8}{*}{$\begin{array}{l}\text { Oreopoulos and } \\
\text { Cahalan (2005); } \\
\text { satellite (MODIS) }\end{array}$} & global & winter & land & liquid & $\tau$ & 85 & 1 & 0.643 \\
\hline & global & winter & land & ice & $\tau$ & 85 & 1 & 0.659 \\
\hline & global & winter & ocean & liquid & $\tau$ & 85 & 1 & 0.700 \\
\hline & global & winter & ocean & ice & $\tau$ & 85 & 1 & 0.686 \\
\hline & global & summer & land & liquid & $\tau$ & 85 & 1 & 0.692 \\
\hline & global & summer & land & ice & $\tau$ & 85 & 1 & 0.678 \\
\hline & global & summer & ocean & liquid & $\tau$ & 85 & 1 & 0.649 \\
\hline & global & summer & ocean & ice & $\tau$ & 85 & 1 & 0.643 \\
\hline \multirow{6}{*}{$\begin{array}{l}\text { Rossow et al. (2002); } \\
\text { satellite (ISCCP) }\end{array}$} & global & both & land & high-level & $\tau$ & 280 & 5 & 0.533 \\
\hline & global & both & ocean & high-level & $\tau$ & 280 & 5 & 0.500 \\
\hline & global & both & land & mid-level & $\tau$ & 280 & 5 & 0.593 \\
\hline & global & both & ocean & mid-level & $\tau$ & 280 & 5 & 0.556 \\
\hline & global & both & land & low-level & $\tau$ & 280 & 5 & 0.525 \\
\hline & global & both & ocean & low-level & $\tau$ & 280 & 5 & 0.552 \\
\hline \multirow{4}{*}{$\begin{array}{l}\text { Pincus et al. (1999); } \\
\text { satellite (FIRE) }\end{array}$} & subtropics & summer & ocean & small cumulus & $\tau$ & 128 & 1 & 0.489 \\
\hline & subtropics & summer & ocean & large cumulus & $\tau$ & 128 & 1 & 0.628 \\
\hline & subtropics & summer & ocean & stratocumulus & $\tau$ & 128 & 1 & 0.486 \\
\hline & subtropics & summer & ocean & stratus & $\tau$ & 128 & 1 & 0.466 \\
\hline
\end{tabular}

${ }^{\star}$ The gridbox sizes stated for the values from Hogan and Illingworth (2003) are the values of $\Delta x$ used in (10); the gridbox size of the raw data they analysed was varied between 2 and $300 \mathrm{~km}$.

${ }^{\dagger}$ Cahalan et al. (1994) used 18 days of data from a microwave radiometer (MWR), hence the very large equivalent gridbox size.

${ }^{\ddagger} \mathrm{Sc}=$ stratocumulus.

bars indicate the differences in gridbox sizes when the wind speed in the cloud level is varied through a reasonable range (5-15 $\mathrm{m} \mathrm{s}^{-1}$ for liquid clouds; $10-20 \mathrm{~m} \mathrm{~s}^{-1}$ for ice clouds). The horizontal error bars on the values from Rossow, et al. (2002) give an indication of the uncertainty in the conversion between the two scaling factors $\chi$ and $\chi_{0}$. The gridbox size scale is logarithmic to account for the large gridbox size used by Cahalan, et al. (1994): 18 days of continuous radar data, which corresponds to a gridbox size of about $15000 \mathrm{~km}$. The relationship between gridbox size $\Delta x$ and fractional standard deviation $f$ derived for ice clouds by Hogan and Illingworth (2003) is shown as the solid grey line, for a wind shear of $5 \mathrm{~m} \mathrm{~s}^{-1} \mathrm{~km}^{-1}$. The attached horizontal error bars indicate the effect of varying shear from 2 to $8 \mathrm{~m} \mathrm{~s}^{-1} \mathrm{~km}^{-1}$. The case-to-case spread in $f$ for their ice clouds, not shown on the graph, is of comparable size in the negative direction, and slightly larger in the positive direction.

Theory predicts that larger gridboxes should contain greater amounts of inhomogeneity (e.g. the results of
Pomroy and Illingworth, 2000), and hence larger values of $f$, but any such dependence is masked by the large differences between the different methods. A dependence of the fractional standard deviation values on the data source, however, is found. More specifically, clouds observed in terms of layer-by-layer water content (shown in Figure 2 as symbols filled in white) have higher values of $f$ on average than those of clouds observed in terms of vertically integrated optical depth or water path (shown as symbols filled in grey); in other words, usually, $f_{w}>f_{\tau}$. This difference is physically realistic. Consider a cloud field that has layers of cloud, each with identical water content statistics. If the inhomogeneities in each layer align perfectly, the optical depth values will be proportional to the water content values in the individual layers, hence $f_{\tau}$ will be exactly the same as $f_{w}$. If the inhomogeneities in the layers do not align exactly, $f_{\tau}$ must be lower than $f_{w}$, as regions of thicker cloud are now aligned with regions of thinner cloud, reducing the occurrence of extreme values and effectively smoothing out the cloud. 


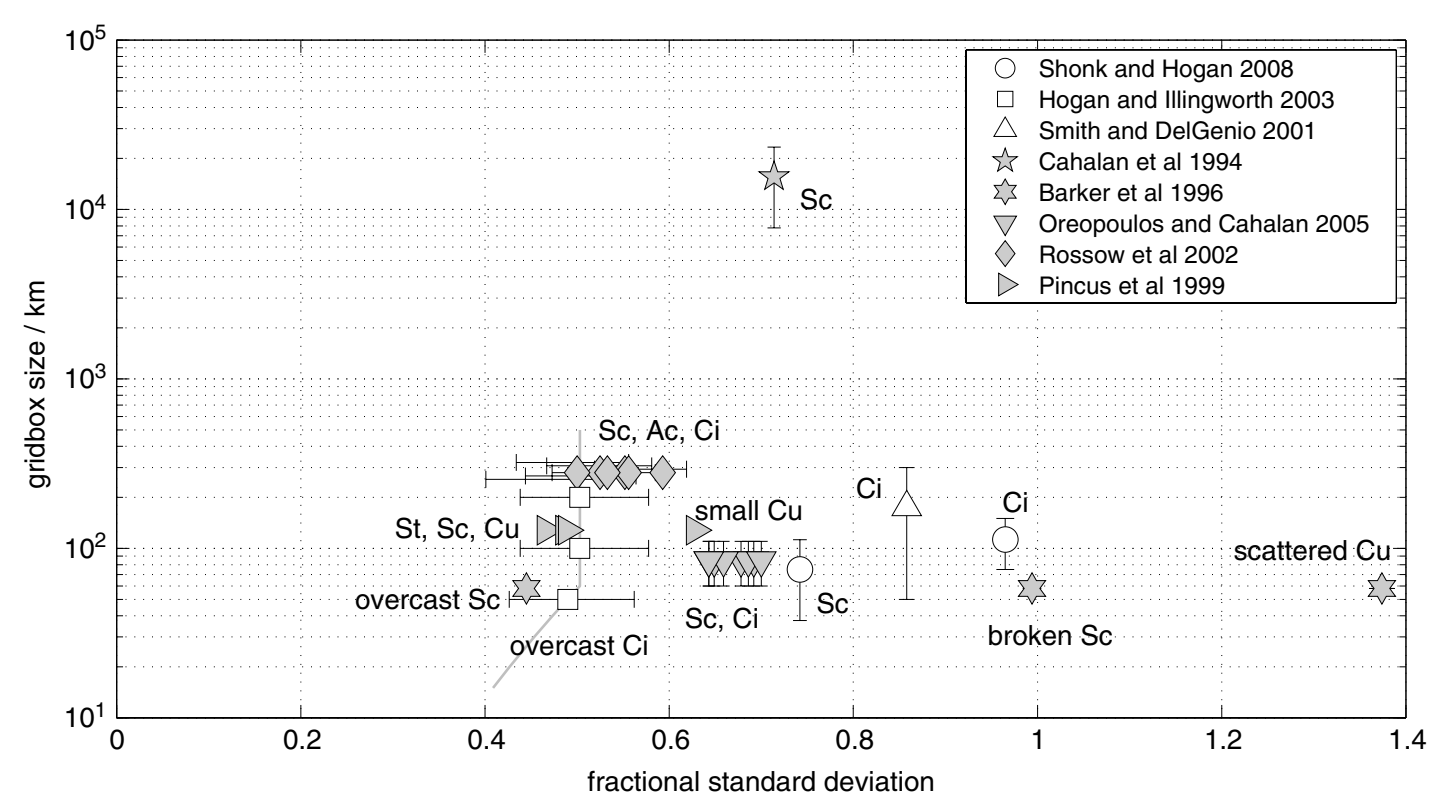

Figure 2. The values of fractional standard deviation $f$, plotted as a function of gridbox size. The shape of each symbol indicates the study from which the value was derived, according to the legend. The white symbols indicate studies that investigated water content; the grey symbols indicate studies that considered water path or optical depth. The types of cloud used in the generation of each value are also shown.

The studies into variability in optical depth used gridboxes with a variety of different cloud covers, and there is a range of $f_{\tau}$ presented. The values found by Barker, et al. (1996) are at the extremes of those found in other studies, with a small value of 0.445 found for overcast stratocumulus, but a very high value of 1.374 for broken cumulus fields. This wide range is most likely caused by the very small size of the pixels in the LandSat data they used: a pixel resolution of order tens of metres. In contrast to this, the studies of Pincus, et al. (1999) and Oreopoulos and Cahalan (2005) used data with a much larger pixel size of $1 \mathrm{~km}$, while Rossow, et al. (2002) used ISCCP satellite data with a pixel size of $5 \mathrm{~km}$. These three studies show much less variability in their values of $f_{\tau}$. The higher measured $f_{\tau}$ of the clouds observed by Barker, et al. (1996) could be caused by LandSat's ability to resolve the contribution to the total variance from smaller scales. In contrast, these small scales cannot be resolved by the lower-resolution sensors used in the studies of Rossow, et al. (2002) and Oreopoulos and Cahalan (2005), with the result that cloud variability is smoothed out across these larger pixels. There could also be a contribution from the approximation $\chi \approx \chi_{0}$, although the size of the horizontal error bars associated with this approximation indicate that this effect is small.

For the studies into water content variability, there is general agreement between the values derived by Smith and DelGenio (2001) and Shonk and Hogan (2008). The equation derived by Hogan and Illingworth (2003), however, gives lower values for all gridbox sizes, implying more homogeneous clouds. This is probably due to the sampling of the data. The scenes evaluated by Shonk and Hogan (2008) consist of a range of different cloud types with various cloud covers, often with whole clouds contained within the gridboxes; those considered by Smith and DelGenio (2001) used horizontal aircraft flights that passed through cloud edges. Hogan and Illingworth (2003) derived their equation using only gridboxes that were entirely cloudy, hence the contributions to variability from cloud edges were not accounted for, perhaps giving a measure of variability that is not applicable to partially cloudy gridboxes. This effect is also seen in the results of Barker, et al. (1996): the fractional standard deviation of scenes of overcast stratocumulus is found to be of a similar value, and much smaller than the corresponding value they found for broken stratocumulus.

Examination of Figure 2 and Table I leads to the conclusion that fractional standard deviations of about 0.75 for water content and 0.65 for water path and optical depth are representative of the variability of all clouds. A calculation of these mean values of $f$, in which the results from each study are equally weighted and the spreads of the values in each study are accounted for, reveals values of $f_{w}=0.75 \pm 0.18$ and $f_{\tau}=0.66 \pm 0.20$. As no notable dependence of fractional standard deviation on any particular category in Table I is discernible, we conclude that these final values can be applied globally.

\subsection{Verification of fractional standard deviation}

This approach to defining the water content values for Tripleclouds is now applied to the 98 cloudy scenes of radar data used by Shonk and Hogan (2008). These scenes are derived from radar, lidar and microwave radiometer measurements made at Chilbolton, and are taken from twelve days of radar data, with a wide variety of different cloud types and shapes present. The scenes are all singlephase: half consist purely of ice cloud, while half consist of lower-level liquid cloud. Each scene contains cloud with a domain mean optical depth of no less than 0.01. We use these data to determine whether the extra approximation of the FSD method has any detrimental effects on the calculated radiation budget, and also to verify our chosen value of $f_{w}$. For each layer of each scene, the Tripleclouds water content values are derived from the mean value in that layer using (2). To examine the dependence of the cloud radiative forcing $(\mathrm{CRF})$ on $f_{w}$, this calculation is performed using values between 0.6 and 1.0. For each $f_{w}$ value, the Edwards-Slingo radiation code (Edwards and Slingo, 1996) is used to calculate the radiative fluxes for each of the radar 
Table II. List of all the cloud representations applied to each of the scenes for this comparison experiment, with their abbreviations.

\begin{tabular}{lll}
\hline $\begin{array}{l}\text { Tripleclouds single- } \\
\text { column representations }\end{array}$ & $\begin{array}{l}\text { TC }(\text { LP16) } \\
\text { TC }\left(\text { FSD } f_{w}\right)\end{array}$ & $\begin{array}{l}\text { Calculation using the LP method, with 16 set as the lower percentile. } \\
\text { Calculation where the lower water content values are defined using } \\
\text { the FSD method, with fractional standard deviation } f_{w} .\end{array}$ \\
\hline
\end{tabular}
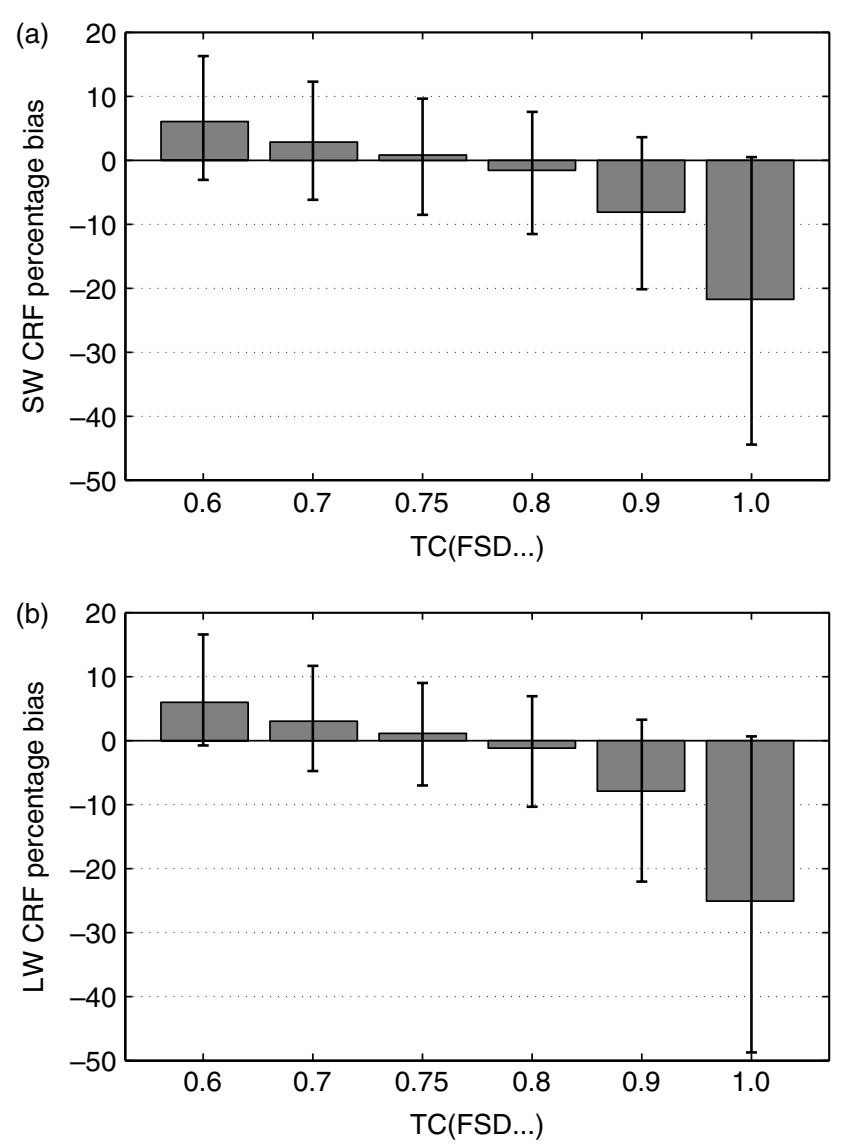

Figure 3. Variation of cloud radiative forcing (CRF) calculated from the radar scenes using the Tripleclouds 'FSD' method for different values of $f_{w}$, as indicated under each bar. Panel (a) shows the short-wave CRF percentage bias; panel (b) shows the long-wave CRF percentage bias. As in Shonk and Hogan (2008), the short-wave CRFs have been inverted in sign to enable easier comparison between the two panels. All biases are with respect to Tripleclouds calculations using the original 'LP' method, with a lower percentile of 16 . The bar height shows the mean bias over the scenes for each $f_{w}$ value, and the error bars show the spread in error bounded by the 10 th and 90 th percentiles.

scenes, and hence the CRF. These calculations using the FSD method are compared with a Tripleclouds calculation using the LP method with the 16th percentile, described in full by Shonk and Hogan (2008), and chosen here as the benchmark calculation. Biases are expressed in terms of top-of-atmosphere (TOA) CRF. As before, the sign of short-wave CRF is inverted so that it is a positive quantity. All calculations use maximum-random overlap. A list of the cloud representations used in this experiment is given in Table II.

The results of these calculations are shown in Figure 3. The form of the plot is the same as the corresponding figures in Shonk and Hogan (2008): the size of each bar indicates the mean value of the bias over the scenes, and the error bars denote the spread in errors for individual scenes in terms of the range spanned by the 10th and 90th percentiles.
As varying $f_{w}$ in the Tripleclouds scheme modulates the degree of inhomogeneity represented, there is a dependence of the CRF biases on the choice of $f_{w}$. Lower values of $f_{w}$ give more homogeneous representations of cloud, hence the biases tend to be more positive; higher $f_{w}$ values give larger degrees of variability, and hence more negative biases. Using a fractional standard deviation of 0.75 gives virtually identical TOA CRFs to the TC(LP16) method.

The spread of the CRF biases with respect to the LP method does appear to be sizeable, although this turns out not to be an issue. If the CRF biases in Figure 3 were to be plotted with respect to ICA calculations, the spreads for the LP and FSD methods turn out to be of comparable size (the FSD spread being slightly larger). The reason for the apparently large spread of biases presented in Figure 3 is that the correlation of biases between the two methods is weak: a larger bias using one method does not imply a larger bias using the other. The slight increase in spread between the LP and FSD methods is inevitable and is most likely caused by variability of the true width of the water content distribution not being captured when using a single value of $f_{w}$. This result verifies our choice of 0.75 for the fractional standard deviation.

\section{Vertical overlap parametrization}

Now we consider vertical overlap and introduce a parametrization of vertical structure that is applicable for use alongside the Tripleclouds scheme, in an attempt to offset the overcompensating biases. It has already been mentioned in section 1 that small changes in global cloud cover result in sizeable biases in the global radiation budget (Randall, et al., 1984; Slingo, 1990). Such changes in global cloud cover can easily be introduced by inaccurate representation of vertical cloud overlap in a GCM. Both Barker, et al. (1999) and $\mathrm{Wu}$ and Liang (2005) used data from a cloud-resolving model (CRM) and calculated the radiative fluxes through the clouds using a radiative transfer code. They both found biases in TOA short-wave flux caused by using random overlap in place of exact overlap that were non-negligible in size with respect to plane-parallel biases. Despite the comparable vertical resolutions of their generated clouds, they found the size of the 'random overlap bias' to be 0.88 (Barker, et al., 1999) and 0.16 (Wu and Liang, 2005) times that of the plane-parallel bias respectively. The smaller ratio reported by $\mathrm{Wu}$ and Liang (2005) was due to the fact that they used 30 days of CRM data in their analysis that contained varying amounts of cloud structure, while Barker, et al. (1999) used just three scenes that were intentionally highly structured.

In this section, we develop and test a vertical overlap parametrization to complement the Tripleclouds scheme that is essentially a modified version of maximum-random overlap. It allows overlap of layers of vertically continuous cloud to vary exponentially depending on the depth of 
the layers with respect to a decorrelation scale. We derive a simple global distribution of the decorrelation scale, and compare the performance of this overlap scheme (referred to as 'exponential-random overlap') with maximum-random overlap.

\subsection{Parametrizations of vertical overlap}

On account of the sensitivity of radiation budget to cloud overlap, it is important that an overlap parametrization is chosen for use with the Tripleclouds scheme that matches real clouds most closely and hence enables them to interact most realistically with atmospheric radiation. It has already been shown that the radiative impact of using an unrealistic cloud overlap approximation can be large. The principal effect of cloud overlap in terms of radiation is to vary total cloud cover in the scene, and changing cloud overlap between minimum, random and maximum has notable effects on radiation budget (Morcrette and Fouquart, 1986). However, these overlap methods are too simplistic to realistically describe cloud arrangements. Geleyn and Hollingsworth (1979) devised a cloud overlap method, known as maximum-random overlap, which remains the most widely used cloud overlap approximation in modern GCMs. Maximum-random overlap applies random overlap to cloud in layers separated by clear sky, but applies maximum overlap to cloud in pairs of layers that are adjacent.

The performance of maximum-random overlap was compared with that of maximum, random and minimum overlap schemes by Tian and Curry (1989), using a dataset derived from aircraft measurements, upper-air soundings, surface and satellite observations. They found that maximum overlap gave the best estimate of cloud cover for vertically continuous cloud, with $81.7 \%$ of the snapshots in their dataset predicting cloud cover to within $2.5 \%$ of the true value, and a systematic overestimation of cloud cover using both random and minimum overlap. For columns with multiple discrete layers of cloud, random overlap was seen to give more realistic representations of cloud cover.

Hogan and Illingworth (2000) used radar data from Chilbolton to derive cloud overlap statistics. They divided two-and-a-half months of data into gridbox-sized domains and calculated cloud cover for pairs of layers using maximum, random and minimum overlap, then compared the values with the true cloud cover of the cloud in these two layers. In agreement with Tian and Curry (1989) they found that, for pairs of cloud layers separated by at least one layer of clear sky, the clouds were overlapped randomly. However, for pairs of layers in vertically continuous cloud, they found that the degree of correlation between the cloud positions decreased with vertical separation of the layers. Also, Barker, et al. (1999) compared the effects of overlap on radiative fluxes using output from a CRM. They compared exact overlap with maximum-random and random overlap, using plane-parallel clouds and evaluating the exitant fluxes and heating rates through the clouds to diagnose performance. With respect to exact overlap, the random overlap case was observed to bias reflected short-wave flux and atmospheric absorption high, and surface absorption low. At a solar zenith angle of $60^{\circ}$, they found outward short-wave flux to be $(75.0 \pm 31.5) \mathrm{W} \mathrm{m}^{-2}$ too high across their three scenes when using random overlap. Implementation of maximum overlap for adjacent layers biased these fluxes in the opposite direction, with short-wave flux biases of $(-58.3 \pm 22.3) \mathrm{W} \mathrm{m}^{-2}$. These results imply that, for pairs of adjacent cloudy layers, maximum overlap results in a combined cloud cover that is too small.

Hogan and Illingworth (2000) introduced an 'overlap parameter', $\alpha$, as a measure of the degree of correlation between cloud positions in a pair of layers. By definition, the cloud cover of the cloud in the two layers when randomly overlapped, $A_{\text {rand }}$, will always be greater than their cloud cover when maximally overlapped, $A_{\max }$. They described the true cloud cover of the two layers, $A$, in terms of these as:

$$
A=\alpha A_{\max }+(1-\alpha) A_{\text {rand }} .
$$

In other words, $\alpha=1$ implies maximum overlap, and $\alpha=0$ implies random overlap. Hence, any cloud cover between those of random and maximum overlap can be obtained in a radiation scheme by setting $\alpha$ to a value between zero and one.

Hogan and Illingworth (2000) went on to plot variation of overlap parameter $\alpha$ with layer vertical separation. For the discontinuous cloud, they found the overlap parameter to be approximately zero for all separations. For continuous cloud, they found a near-exponential decrease of the overlap parameter with separation. They then described overlap parameter between such layers in terms of a decorrelation height $Z_{0 \alpha}$ according to the equation:

$$
\alpha=\exp \left(-\frac{\Delta z}{Z_{0 \alpha}}\right),
$$

where the exact value of $Z_{0 \alpha}$ is related to the vertical resolution and the horizontal domain size. The mathematical basis of this result was considered by Astin and DiGirolamo (2006). They found the exponential decay of $\alpha$ with height to apply when the distribution of cloud layer thickness was also exponential.

Since its initial suggestion, a number of other studies have made use of overlap parameter $\alpha$ (e.g. Bergman and Rasch, 2002; Pincus, et al., 2005). Mace and Benson-Troth (2002) applied the algorithm of Hogan and Illingworth (2000) to radar data from the Atmospheric Radiation Measurement (ARM) programme, which has radars at several latitudes. They found that the value of decorrelation height varied with location, and also with season at the midlatitude Great Plains site. In the summer, an increase in upright convection here was found to lead to more maximally overlapped clouds and larger decorrelation scales. Naud, et al. (2008) also used cloud radar data from ARM in combination with reanalysis data from the National Centers for Environmental Prediction (NCEP) to investigate the effect of changes in vertical motion, convective stability and wind shear on the decorrelation height. Again using the method of Hogan and Illingworth (2000), they found vertically continuous clouds to be more maximally overlapped in the presence of vertical motion in midlatitudes and decreased convective stability in the Tropics. These results are intuitive, as both these conditions result in clouds that increase in height more quickly, giving clouds that are nearer vertical. Increases in wind shear were found to increase the randomness of the overlap, with overlap becoming less than random for large separations in some cases.

In other words, the optimum value of decorrelation height across the world is likely to be strongly dependent 


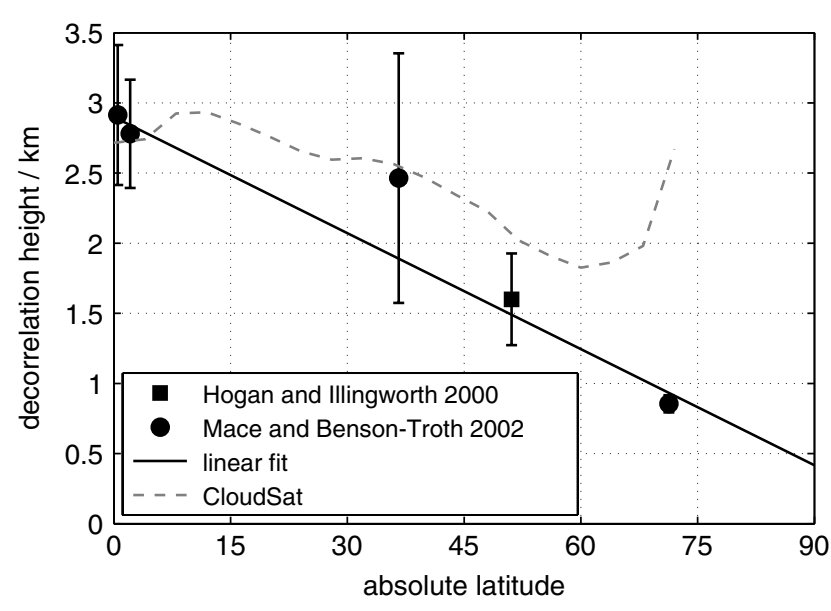

Figure 4. Fit of decorrelation height $Z_{0 \alpha}$ as a function of latitude, using values derived from the studies of Hogan and Illingworth (2000) and Mace and Benson-Troth (2002). The fitted line is shown as black solid, and the dashed line shows a series of decorrelation heights calculated using data from CloudSat and CALIPSO.

on location. Using the information from the studies of Hogan and Illingworth (2000) and Mace and Benson-Troth (2002), we are in a position to derive a simple relationship between decorrelation height and latitude. The values of decorrelation height are plotted in Figure 4 as a function of absolute latitude for each of the values reported in the two papers. The points indicate the mean value of decorrelation height; the error bars show the spread at each location.

Our plot includes a series of values for decorrelation height derived as a function of absolute latitude from satellite radar data from CloudSat and lidar data from CALIPSO (the grey dashed line). This series is calculated using values of $\alpha$ according to the calculations of Mace, et al. (2009), and appears to show a more maximally overlapped scenario than the values derived from surface radar sites. We attribute this to a combination of two effects. Firstly, the vertical resolution and gridbox size used to calculate $\alpha$ from the data are both larger, which will result in larger decorrelation heights (Table I of Hogan and Illingworth, 2000 and Table III later in this study). Secondly, the inclusion of rain in the analysis would artificially cause the overlap to be more maximum. Barker (2008a) found that inclusion of precipitation could cause an increase in decorrelation height of order $500 \mathrm{~m}$. (All of the surface radar studies identified any rain regions using a lidar ceilometer and removed them from the data.) Barker (2008b) also obtained a continuous distribution of decorrelation height with latitude from CloudSat and CALIPSO data. He found a much weaker relationship between the two, with a value not varying much from $2 \mathrm{~km}$, although his method of deriving decorrelation height is different from the other studies.

A simple linear fit is calculated that relates $Z_{0 \alpha}$ in kilometres to the absolute latitude $\phi$ in degrees. On account of the inclusion of rain, the data from CloudSat is excluded. The linear fit is found to be:

$$
Z_{0 \alpha}=2.899-0.02759 \phi \text {. }
$$

A latitude-dependent decorrelation height has recently been implemented into the ECMWF model, based on this result (Jean-Jacques Morcrette, personal communication). The decorrelation lengths are calculated via a smoothed version of (13) and used in the implementation of McICA.
We recognise that this equation is crude in certain respects: we have assumed a linear fit based on only five points, and not taken into account any dependence of decorrelation height on either the presence of convection or the effects of wind shear. However, such a simple equation should be sufficient to investigate the radiative effect of changing overlap from maximum-random to something more realistic. The values taken from the studies are calculated as an average for each location, with error bars indicating the uncertainty in decorrelation height that could partially be due to neglecting these effects. The radiative effect of this uncertainty on the performance of exponentialrandom overlap is investigated in Part II.

\subsection{The beta overlap parameter}

In this subsection, we describe how exponential-random overlap can be implemented simply into a radiation code. We allow overlap between adjacent layers to vary between maximum and random according to an overlap parameter like that of Hogan and Illingworth (2000). However, while their $\alpha$ succinctly describes overlap for a system with two regions in each layer, its definition becomes ambiguous when a third region is added. For this reason, we show how an alternative definition of overlap parameter, $\beta$, can make the implementation of exponential-random overlap for multiple regions much easier. Overlap between a pair of adjacent layers is then determined via a decorrelation height according to (13). It should also be noted that overlap is only calculated between adjacent layers using the decorrelation height, not at a distance as in Hogan and Illingworth (2000).

To describe how overlap with $\beta$ is implemented, we must first re-introduce the concept of an overlap matrix. This was introduced by Shonk and Hogan (2008), and is a mathematical representation of the overlap of clouds in an arbitrary pair of layers. The two-region and three-region overlap matrices are given by:

$$
\begin{aligned}
& \mathbf{O}=\left(\begin{array}{cc}
A_{\mathrm{c}, \mathrm{c}} & A_{\mathrm{c}, \mathrm{l}} \\
A_{\mathrm{l}, \mathrm{c}} & A_{\mathrm{l}, \mathrm{l}}
\end{array}\right), \\
& \mathbf{O}=\left(\begin{array}{lll}
A_{\mathrm{c}, \mathrm{c}} & A_{\mathrm{c}, \mathrm{l}} & A_{\mathrm{c}, \mathrm{h}} \\
A_{\mathrm{l}, \mathrm{c}} & A_{1,1} & A_{\mathrm{l}, \mathrm{h}} \\
A_{\mathrm{h}, \mathrm{c}} & A_{\mathrm{h}, \mathrm{l}} & A_{\mathrm{h}, \mathrm{h}}
\end{array}\right),
\end{aligned}
$$

where $A_{x, y}$ denotes the fractional area of the domain with region $x$ in the upper layer and region $y$ in the lower layer. As in Shonk and Hogan (2008), the subscripts c, 1 and h are used to denote the clear-sky region, the cloudy region with lower water content and the cloudy region with higher water content respectively. For the two-region case, the single cloudy region is denoted by 1 .

\subsubsection{Beta with one cloudy region}

As for the overlap parameter $\alpha$ of Hogan and Illingworth (2000), our new overlap parameter $\beta$ has a value of zero for a pair of layers that are overlapped randomly and a value of one for a pair that are overlapped maximally, and can have any intermediate value. Consider a pair of adjacent layers in an idealised two-region cloud scene, with one region representing the cloud and the other representing the clear sky, as shown in Figure 5. The pair of partially cloudy layers has two different cloud fractions, which are denoted as $L$ 
(a) $\beta_{\mathrm{c}}=\beta_{\mathrm{l}}=0.0$

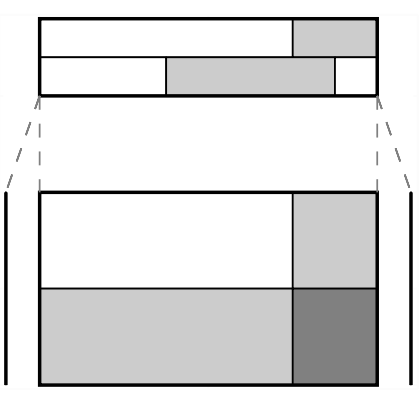

(b) $\beta_{\mathrm{c}}=\beta_{\mathrm{I}}=0.5$

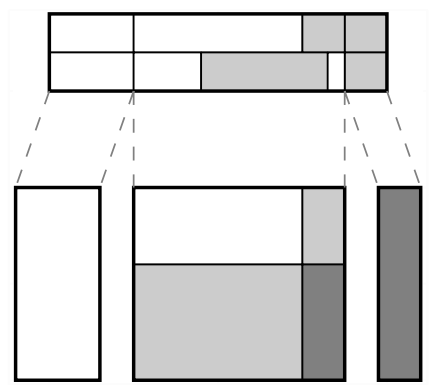

(c) $\beta_{\mathrm{c}}=\beta_{\mathrm{I}}=1.0$

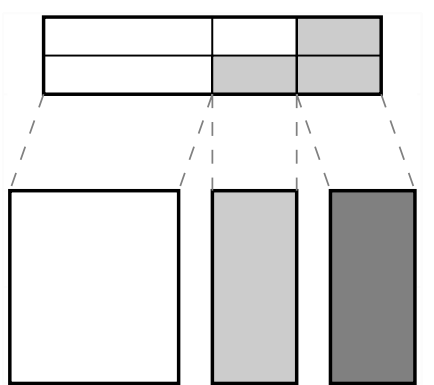

Figure 5. A schematic showing the effects of the overlap parameter $\beta$ for a pair of cloudy layers with cloud fractions of 0.25 and 0.5 in the upper and lower layers respectively. Each panel shows the alignment of the clouds using a different value of $\beta$. The same value of $\beta$ is applied to both the clear-sky and cloudy regions. The top part of each panel shows the clouds viewed from the side; the bottom part shows the clouds viewed from above. See the text for further explanation.

and $l$, with $L$ referring to the larger of the two values. The corresponding clear-sky fractions are denoted as $C$ and $c$, again with $C$ referring to the larger of the two values. Overlap parameter $\beta$ for a given region $x$ defines the area of that region that is overlapped maximally, $m_{x \text {,true }}$, as a fraction of the maximum possible maximally overlapped area, $m_{x, \max }$ :

$$
\beta_{x}=\frac{m_{x, \text { true }}}{m_{x, \max }} .
$$

First, the maximally overlapped $\mathbf{M}$ matrix is calculated. In the case of random overlap (Figure 5(a)), none of the regions are overlapped maximally and $\beta=0$; in the case of maximum overlap (Figure 5(c)), similar regions in the two layers are overlapped as much as is possible and $\beta=1$. In other words, the maximum overlap matrix is written as:

$$
\mathbf{M}=\left(\begin{array}{cc}
\beta c & 0 \\
0 & \beta l
\end{array}\right)
$$

The random overlap stage is then performed. Any remaining clear sky or cloud in either layer that has not been overlapped maximally is overlapped assuming no correlation between the cloud positions. One way of depicting this is to align the regions in the two layers orthogonally and view them from above, as shown in the middle boxes on the bottom rows of each of the panels. This gives a random overlap matrix of:

$$
\begin{aligned}
& \mathbf{R}= \\
& \frac{1}{(1-\beta c-\beta l)}\left(\begin{array}{cc}
(C-\beta c)(c-\beta c) & (C-\beta c)(L-\beta l) \\
(l-\beta l)(c-\beta c) & (l-\beta l)(L-\beta l)
\end{array}\right) .
\end{aligned}
$$

The total overlap matrix $\mathbf{O}$ is then found by summing these two matrices; in other words, $\mathbf{O}=\mathbf{M}+\mathbf{R}$.

It can also be shown that, for a two-region system where the $\beta$ values are the same for both the clear sky and the cloud, the two overlap parameters can be related to each other via an equation in terms of the difference in cloud fraction in the pair of layers, $\Delta L=L-l$ :

$$
\alpha=\beta+\frac{(1-\beta) \Delta L}{\Delta L+(1 / \beta-1)},
$$

which leads to the result that $\alpha=\beta$ for cases where $\Delta L=0$ and $\alpha>\beta$ for cases where $\Delta L>0$.

\subsubsection{Beta with two cloudy regions}

When implementing a second cloudy region into each layer, such as in the Tripleclouds scheme, the advantages of using $\beta$ in place of $\alpha$ to describe overlap become apparent. It is straightforward to extend the method described in the previous subsection and Figure 5 to account for an extra cloudy region that is overlapped according to its own value of $\beta$. A key advantage of using $\beta$ for a three-region system is that its values may be different for the regions of clear sky and cloud. For a system with any number of regions, the values of $\beta$ for each region may be independently selected as any combination of numbers in the interval zero to one. The values of $\alpha$ for each region must be dependent on one another. In a two-region system with cloud and clear sky, it is intuitive to set the two decorrelation heights to be the same, implying that $\beta_{\mathrm{c}}=\beta_{1}$, hence $Z_{0 \beta \mathrm{c}}=Z_{0 \beta 1}$. For a Tripleclouds representation, however, it is better to set the two 'in-cloud' overlap parameters, $\beta_{1}$ and $\beta_{\mathrm{h}}$, equal to each other but to allow the 'cloud-boundary' overlap parameter $\beta_{\mathrm{c}}$ to be a separate value.

A typical ratio of cloud-boundary and in-cloud decorrelation scales was found by Räisänen, et al. (2004). They used cloud fields derived from a GCM simulation that applied a CRM to each grid column, following the work of Khairoutdinov and Randall (2001). For their CRM data, they determined the ratio of cloud-boundary to in-cloud decorrelation height to be approximately equal to 2 for pairs of adjacent layers. It should be borne in mind that their definition of in-cloud decorrelation length $L_{\mathrm{cw}}$ is different from our $Z_{0 \beta 1}$ : ours is determined using in-cloud overlap parameter $\beta_{1}$; theirs uses rank correlation of the subgrid-scale distribution of water content in the two layers. However, it can be shown that, if that distribution is a two-point distribution function, as used in Tripleclouds, the overlap parameter and the rank correlation coefficient are identical in cases where either layer has a cloud fraction of 1 ; in which case, $Z_{0 \beta 1}=L_{\mathrm{cw}}$. Hogan and Illingworth (2003) also evaluated in-cloud decorrelation height in terms of correlation coefficient, and found it to take values of order 0.5 to $1.0 \mathrm{~km}$ for domain sizes comparable to those used by Hogan and Illingworth (2000). If a typical average wind speed of $20 \mathrm{~m} \mathrm{~s}^{-1}$ is used to convert the temporal domain sizes of Hogan and Illingworth (2000) to physical sizes, the ratio of cloud-boundary to in-cloud decorrelation heights turns out to be about 2. Despite the fact that these previous studies have not used exactly the same measures of 


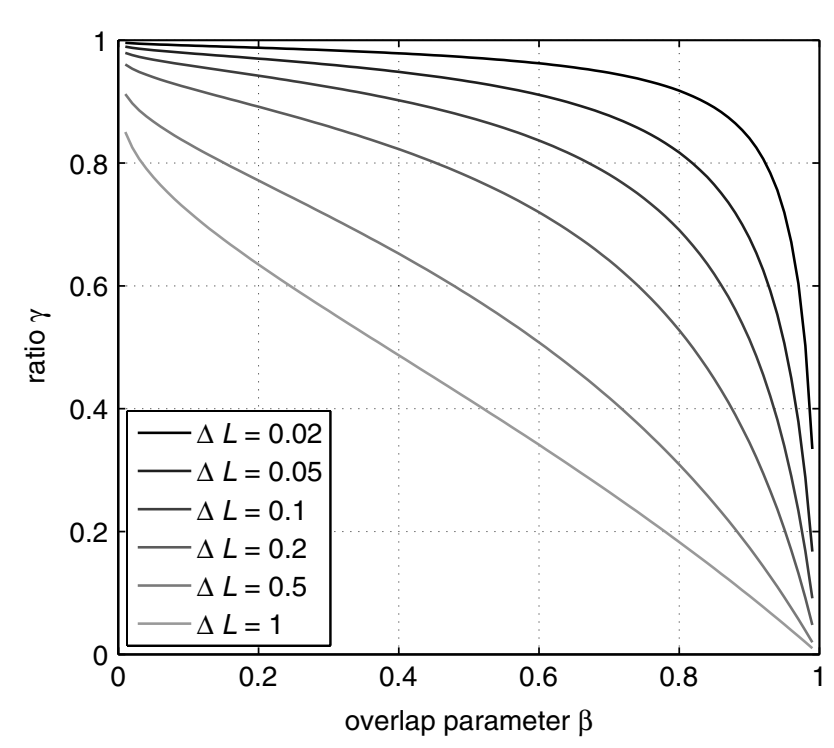

Figure 6. Ratio $\gamma$ of the decorrelation heights determined from $\alpha$ and $\beta$ in (19) for different values of cloud fraction difference $\Delta L$.

decorrelation scale, they are sufficiently close to support our use of $Z_{0 \beta \mathrm{c}}=2 Z_{0 \beta 1}=2 Z_{0 \beta \mathrm{h}}$ for our three-region scheme. Additionally, it was found by Barker (2008b) and will be shown in Part II that calculated fluxes are fairly insensitive to exact value of decorrelation height over the range that we use here.

\subsection{Decorrelation heights in terms of alpha and beta}

The fact that $\alpha \neq \beta$ in most cases implies that we cannot assume the height over which $\alpha$ decorrelates, $Z_{0 \alpha}$, to be equal to the equivalent height for $\beta, Z_{0 \beta}$. In other words, our global decorrelation height distribution (13) cannot be applied to the clouds with $Z_{0 \beta}$ in place of $Z_{0 \alpha}$. In this section, we address the issue of the difference between the two parameters by seeking a relationship between the two decorrelation height scales, $Z_{0 \alpha}$ and $Z_{0 \beta}$. Throughout, we use terminology that applies to a two-region system. Our approach is to investigate the ratio of the two decorrelation heights and identify whether there is a single value of this ratio. We refer to the ratio as $\gamma$, and define it as:

$$
\gamma=\frac{Z_{0 \beta}}{Z_{0 \alpha}}
$$

Figure 6 shows $\gamma$ as a function of overlap parameter $\beta$ for a two-region system, calculated from (19) for several values of $\Delta L$. Only in three cases is it true that $Z_{0 \alpha}=Z_{0 \beta}$ : if $\beta=0$; if $\beta=1$; or if the cloud fraction is the same in both layers.

We now perform an experiment to determine a value for $\gamma$. Eighteen months of continuous data from the $94 \mathrm{GHz}$ vertically pointing radar at Chilbolton are used, in the period from 01 April 2003 to 30 September 2004. The raw data are stored at $60 \mathrm{~m}$ vertical resolution and $30 \mathrm{~s}$ temporal resolution. Each day of data is divided into a number of scenes of a given temporal length $\Delta t$, and averaged vertically to give a particular vertical resolution $\Delta z$. These resolutions are chosen to match those used by Hogan and Illingworth (2000). Periods with data missing or precipitation present are excluded from the investigation. Precipitation is identified using data from a lidar ceilometer, which locates cloud base and can hence determine whether precipitation is present
Table III. Values of overlap ratio $\gamma$ calculated from cloud radar data in the period from 01 April 2003 to 30 September 2004, for different horizontal and vertical resolutions, $\Delta t$ and $\Delta z$.

\begin{tabular}{lrccc}
\hline$\Delta t(\min )$ & $\Delta z(\mathrm{~m})$ & $Z_{0 \alpha}(\mathrm{km})$ & $Z_{0 \beta}(\mathrm{km})$ & $\gamma$ \\
\hline 20 & 360 & 1.491 & 1.102 & 0.739 \\
& 720 & 1.857 & 1.448 & 0.780 \\
& 1080 & 2.239 & 1.797 & 0.802 \\
60 & 360 & 1.619 & 1.199 & 0.741 \\
& 720 & 1.934 & 1.505 & 0.778 \\
& 1080 & 2.241 & 1.803 & 0.805 \\
180 & 360 & 1.653 & 1.262 & 0.763 \\
& 720 & 2.157 & 1.672 & 0.775 \\
& 1080 & 2.493 & 1.947 & 0.781 \\
\hline
\end{tabular}

using the same technique as Illingworth, et al. (2007). For each pair of adjacent layers in each scene, the cloud fractions in the upper and lower layer are stored, as well as the combined cloud cover for that pair, $A$. A range of values of $Z_{0 \alpha}$ and $Z_{0 \beta}$ are then applied to the cloud fractions, giving 'predicted' cloud covers $A_{\alpha}$ and $A_{\beta}$, also stored for each pair of layers in each scene. The optimal value of decorrelation height is then determined as the value where the predicted mean cloud cover over all scenes and layer pairs equals the true mean cloud cover over all scenes and layer pairs $\left(\bar{A}_{\alpha}=\bar{A}\right.$ or $\left.\bar{A}_{\beta}=\bar{A}\right)$. Overlap ratio $\gamma$ is then determined by dividing the two scales. It should be noted that the method used here to determine decorrelation height is different from the methods used to derive the values of $Z_{0 \alpha}$ presented in Figure 4. However, the aim of this subsection is to identify the ratio of decorrelation heights, and we are using the same method to determine both $Z_{0 \alpha}$ and $Z_{0 \beta}$. We make the assumption that the ratio we derive here from the 'effective' decorrelation heights will also be applicable to decorrelation heights calculated using a exponential least-squares fit (as Hogan and Illingworth, 2000 and Mace and Benson-Troth, 2002).

The overlap ratio is found to have a dependence on both vertical resolution and horizontal domain size, with a range of values as shown in Table III. We now select a value for use with the Tripleclouds scheme. As it will be applied to a range of data types, and as the conversion of the horizontal temporal domain size to a physical size is so imprecise, we select a typical value of 0.75 . When applied to (13), this becomes:

$$
Z_{0 \beta}=2.174-0.0207 \phi
$$

When a second cloudy region is introduced, this equation gives the cloud-boundary decorrelation height $Z_{0 \beta \mathrm{c}}$. The in-cloud decorrelation height $Z_{0 \beta 1}$ is set to half of this value, and applied to both cloud regions $\left(Z_{0 \beta 1}=Z_{0 \beta \mathrm{h}}\right)$.

\subsection{Comparison of overlap methods}

An experiment is now performed to compare the effects of overlap on the various cloud representations in terms of their interactions with atmospheric radiation. For this experiment, we make use of the same 98 cloudy scenes used in section 2.3. Random overlap $(\beta=0)$ and maximumrandom overlap $(\beta=1)$ are applied, along with five 
Table IV. List of all the cloud representations performed on each of the scenes for the overlap comparison experiment, with their abbreviations. All Tripleclouds calculations are performed using the LP method.

\begin{tabular}{lll}
\hline $\begin{array}{l}\text { Tripleclouds independent- } \\
\text { column representations }\end{array}$ & TCICA & $\begin{array}{l}\text { Control calculation on raw data with the Tripleclouds } \\
\text { scheme applied to each layer. }\end{array}$ \\
\hline $\begin{array}{l}\text { Tripleclouds single- } \\
\text { column representations, }\end{array}$ & TC $(\mathrm{LP} 16) \mathrm{r}$ & Calculation using random overlap. \\
using a lower percentile of 16 & TC $(\mathrm{LP} 16) \mathrm{m}$ & Calculation using maximum-random overlap. \\
& & Calculation using exponential-random overlap with \\
& $Z_{0 \beta \mathrm{c}}(\mathrm{km})$ determined from $Z_{0 \alpha}$ using a certain $\gamma$ value.
\end{tabular}

calculations using exponential-random overlap. The latitude at Chilbolton is $51.1^{\circ} \mathrm{N}$, implying that $Z_{0 \alpha}=1.489 \mathrm{~km}$, according to (13). The exponential-random calculations vary $\gamma$ from 0.55 to 0.95 in steps of 0.1 , which has the effect of varying decorrelation height $Z_{0 \beta \mathrm{c}}$ from $0.82 \mathrm{~km}$ to $1.42 \mathrm{~km}$, using (20).

To independently investigate the effects of modifying the vertical overlap, we revert to using the lower percentile (LP) method for Tripleclouds described in Shonk and Hogan (2008), using the 16th percentile to determine the pairs of water content values. This removes all compensations to errors and spread introduced by the FSD method. Furthermore, we use Tripleclouds ICA calculations as our benchmark. The Tripleclouds approximation is applied to each layer of the high-resolution radar data, which is then evaluated in a column-by-column approach, resulting in exact overlap. Shonk and Hogan (2008) showed that the ICA was a very good approximation to a single-column integration with Tripleclouds where overlap was conserved. The short-wave and long-wave CRF biases are compared, with the sign of the short-wave CRFs changed again for ease of comparison with the long-wave CRFs. A full list of the cloud representations used in this experiment, along with all of the abbreviations, is presented in Table IV.

The results of this experiment are presented in Figure 7. The height of the bars corresponds to the mean bias over the 98 cloudy scenes, and the error bars show the range spanned by the 10th and 90th percentiles of the distribution, hereafter referred to as the 'spread'. We see a more positive bias for random overlap and a more negative bias for maximumrandom overlap, in agreement with Barker, et al. (2003) and Stephens, et al. (2004), among others. The smaller values of decorrelation height give biases nearer those of random overlap, while the higher values of $Z_{0 \beta c}$ behave in a more maximum-random manner. It is also seen that there is no significant increase in error spread when comparing exponential-random overlap with maximum-random.

In terms of both short-wave and long-wave CRF, a value of $\gamma=0.75$ for the decorrelation height calculation produces small biases, although it is seen that the sensitivity of the CRF biases to $\gamma$ is small. As before, there is a slight difference in biases for the two spectral regions, with a more positive bias in the long-wave than in the short-wave. In fact the difference in bias over the range of $\gamma$ values shown is smaller than the slight positive bias observed in the long-wave CRF, suggesting that the exact choice of $\gamma$ is somewhat arbitrary within the range used here. However, attempting to obtain an optimum value of $\gamma$ from these results is imperfect: while we calculated $\gamma$ from data at Chilbolton, the equation that defines the decorrelation height was calculated as a fitted line to global data. In other words, the error introduced by varying $\gamma$ is smaller than the error introduced by uncertainty
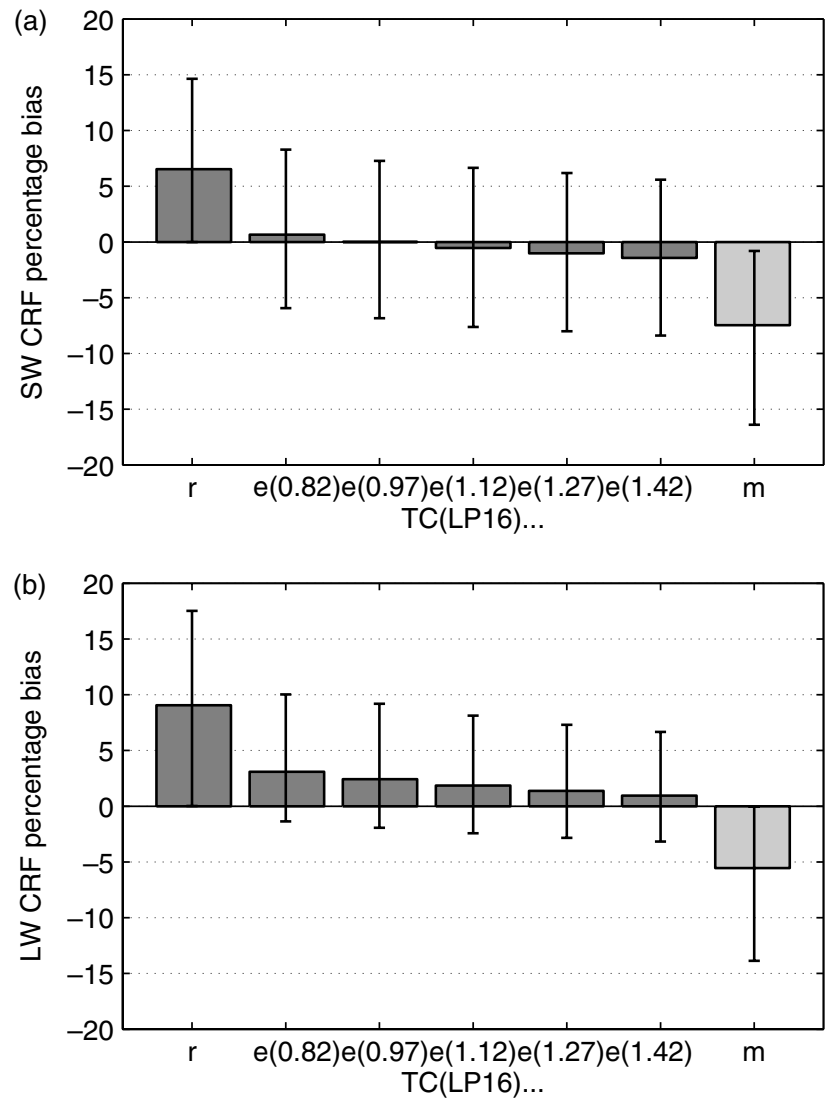

Figure 7. Variation of the cloud radiative forcing calculated from the scenes using different cloud overlap schemes: (a) the short-wave CRF percentage bias with respect to the TCICA CRFs, and (b) the long-wave CRF percentage bias. Short-wave CRFs are inverted in sign. All calculations use Tripleclouds with a lower percentile of 16, TC(LP16); the overlap scheme for each bar is indicated underneath using the abbreviations in Table IV. The bars show the mean bias over the scenes for each overlap scheme, and the error bars show the spread in error bounded by the 10th and 90th percentiles for each scheme.

in the position of the fitted line in Figure 4. These results do show that exponential-random overlap does account for much of the overcompensation caused by maximumrandom overlap, and suggest that (21) may be globally applicable.

\section{Discussion and conclusions}

Misrepresentation of cloud structure is recognised as a potential cause of significant biases in radiation calculations. These biases originate from two sources: use of horizontally homogeneous, plane-parallel clouds; and use of vertical overlap schemes that assume clouds in adjacent model layers are maximally overlapped. This study aimed to combine the 
Tripleclouds scheme of Shonk and Hogan (2008) with the idea of cloud overlap that decorrelated with height according to some length-scale (Hogan and Illingworth, 2000) in a method that could be easily applied in operational radiation schemes. The combination of the two parametrizations could eliminate biases arising from poor horizontal and vertical cloud structure representation.

The initial investigations into Tripleclouds by Shonk and Hogan (2008) calculated the two water content values for a layer of a gridbox using the 'lower percentile method', where the exact water content distribution for that layer was known and the lower value fixed to the 16th percentile. Here, we defined an alternative 'fractional standard deviation' method that does not require knowledge of an exact distribution, and uses a fixed fractional standard deviation of water content, $f_{w}$. A review of a number of studies showed that there is no consensus on how it varies with cloud type, gridbox size and so on. A globally representative value of this quantity was found to be $0.75 \pm 0.18$. Testing of the method on radar data using the Edwards-Slingo radiation code showed 0.75 to be the most appropriate value for $f_{w}$. Calculations using this value gave almost zero bias with respect to a calculation that uses the lower percentile method, but with a small increase in spread.

A parametrization of vertical overlap was then developed from the results of Hogan and Illingworth (2000), that describes overlap in terms of the height-scale over which overlap parameter $\alpha$ decorrelates, $Z_{0 \alpha}$. Using their results in combination with those of Mace and Benson-Troth (2002), a distribution of $Z_{0 \alpha}$ with latitude was determined that could be applied to global model data. An alternative description of overlap parameter was introduced, referred to as $\beta$, that can more conveniently and intuitively describe overlap than $\alpha$ in a three-region system. A relationship between $Z_{0 \alpha}$ and its $\beta$ equivalent, $Z_{0 \beta}$, was sought, again using radar data. An average value of their ratio, referred to as $\gamma$, was found to be 0.75 , enabling us to convert our $Z_{0 \alpha}$ equation to $Z_{0 \beta}$. The overlap scheme, referred to as 'exponential-random overlap', was then compared with other overlap schemes using the same sets of radar data and the same method. With respect to exact overlap, both random and maximumrandom overlap gave radiative biases of between $6 \%$ and $9 \%$ in both short-wave and long-wave cloud radiative forcing. Biases were reduced to much less than $1 \%$ in the shortwave, and just over $1 \%$ in the long-wave, with no significant increase in spread.

We have shown here independently that the two components of the full Tripleclouds scheme can drastically reduce the biases in cloud radiative forcing introduced by misrepresentation of cloud structure. The next stage is to perform radiative transfer calculations using global model data to evaluate the global effect of the horizontal and vertical components of the Tripleclouds scheme. This will both investigate the performance of the Tripleclouds scheme with respect to the plane-parallel maximum-random representation, and also consider the separate effects of the horizontal and vertical components on global radiation budget. Part II of this paper describes these experiments.

\section{Acknowledgements}

We thank Keith Shine and Jean-Jacques Morcrette for their thoughts and input to this study. This research was funded by a Research Endowment Trust Fund studentship at the University of Reading and NERC grant NE/F011261/1.

\section{References}

Astin I, DiGirolamo L. 2006. The relationship between $\alpha$ and the crosscorrelation of cloud fraction. Q. J. R. Meteorol. Soc. 132: 2475-2478.

Barker HW 1996. A parametrisation for computing grid-averaged solar fluxes for inhomogeneous marine boundary layer clouds. Part I: Methodology and homogeneous biases. J. Atmos. Sci. 53: 2289-2303.

Barker HW. 2008a. Overlap of fractional cloud for radiation calculations in GCMs: A global analysis using CloudSat and CALIPSO data. J. Geophys. Res. 113: DOI: 10.1029/2007JD009677.

Barker HW. 2008b. Representing cloud overlap with an effective decorrelation length: An assessment using CloudSat and CALIPSO data. J. Geophys. Res. 113: DOI: 10.1029/2008JD010391.

Barker HW, Davies JA. 1992. Solar radiative fluxes for stochastic, scale-invariant broken cloud fields. J. Atmos. Sci. 49: 1115-1126.

Barker HW, Wielicki BA, Parker L. 1996. A parametrisation for computing grid-averaged solar fluxes for inhomogeneous marine boundary layer clouds. Part II: Validation using satellite data. J. Atmos. Sci. 53: 2304-2316.

Barker HW, Stephens GL, Fu Q. 1999. The sensitivity of domain-averaged solar fluxes to assumptions about cloud geometry. Q. J. R. Meteorol. Soc. 125: 2127-2152.

Barker HW, Stephens GL, Partain PT, Bergman JW, Bonnel B, Campana K, Clothiaux EE, Clough S, Cusack S, Delamere J, Edwards J, Evans KF, Fouquart Y, Freidenreich S, Galin V, Hou Y, Kato S, Li J, Mlawer E, Morcrette J-J, O'Hirok W, Räisänen P, Ramaswamy V, Ritter B, Rozanov E, Schlesinger M, Shibata K, Sporyshev P, Sun Z, Wendisch M, Wood N, Yang F. 2003. Assessing 1D atmospheric solar radiative transfer models: Interpretation and handling of unresolved clouds. J. Climate 16: 2676-2699.

Bergman JW, Rasch PJ. 2002. parametrising vertically coherent cloud distributions. J. Atmos. Sci. 59: 2165-2182.

Cahalan RF, Ridgway W, Wiscombe WJ, Bell TL, Snyder JB. 1994. The albedo of fractal stratocumulus clouds. J. Atmos. Sci. 51: 2434-2455.

Cairns B, Lacis AA, Carlson BE. 2000. Absorption within inhomogeneous clouds and its parametrisation in general circulation models. J. Atmos. Sci. 57: 700-714.

Carlin B, Fu Q, Lohmann U, Mace GG, Sassen K, Comstock JM. 2002. High-cloud horizontal inhomogeneity and solar albedo bias. J. Climate 15: 2321-2339.

Colman R. 2003. A comparison of climate feedbacks in general circulation models. Clim. Dyn. 20: 865-873.

Davis A, Gabriel PM, Lovejoy S, Schertzer D, Austin G. 1990. Discrete angle radiative transfer. Part III: Numerical results and applications. J. Geophys. Res. 95: 11729-11742.

Edwards JM, Slingo A. 1996. Studies with a flexible new radiation code. I: Choosing a configuration for a large-scale model. Q. J. R. Meteorol. Soc. 122: 689-719.

Geleyn JF, Hollingsworth A. 1979. An economical, analytical method for the computation of the interaction between scattering and line absorption of radiation. Contrib. Atmos. Phys. 52: 1-16.

$\mathrm{Gu}$ Y, Liou K. 2006. Cirrus cloud horizontal and vertical inhomogeneity effects in a GCM. Meteorol. Atmos. Phys. 91: 223-235.

Hogan RJ, Illingworth AJ. 2000. Deriving cloud overlap statistics from radar. Q. J. R. Meteorol. Soc. 126: 2903-2909.

Hogan RJ, Illingworth AJ. 2003. parametrising ice cloud inhomogeneity and the overlap of inhomogeneities using cloud radar data. J. Atmos. Sci. 60: 756-767.

Illingworth AJ, Hogan RJ, O'Connor EJ, Bouniol D, Brooks ME, Delanoë J, Donovan DP, Gaussiat N, Goddard JWF, Haeffelin M, Klein Baltink H, Krasnov OA, Pelon J, Piriou J-M, Protat A, Russchenberg HWJ, Seifert A, Tompkins AM, Van Zadelhoff G-J, Vinit F, Willén U, Wilson DR, Wrench CL. 2007. Cloudnet - Continuous evaluation of cloud profiles in seven operational models using ground-based observations. Bull. Amer. Meteorol. Soc. 88: 883-898.

Khairoutdinov MF, Randall DA. 2001. A cloud-resolving model as a cloud parametrization in the NCAR Community Climate Model: preliminary results. Geophys. Res. Lett. 28: 3617-3620.

Mace GG, Benson-Troth S. 2002. Cloud-layer overlap characteristics derived from long-term cloud radar data. J. Climate 15: 2505-2515.

Mace GG, Zhang Q, Vaughan M, Marchand R, Stephens GL, Trepte C, Winker D. 2009. A description of hydrometeor layer occurrence statistics derived from the first year of merged CloudSat and CALIPSO data. J. Geophys. Res. 114: DOI: 10.1029/2007JD009755. 
Morcrette J-J, Fouquart Y. 1986. The overlapping of cloud layers in short-wave radiation parametrisations. J. Atmos. Sci. 43: 321-328.

Morcrette J-J, Barker HW, Cole JNS, Iacono MJ, Pincus R. 2008. Impact of a new radiation package, McRad, in the ECMWF Integrated Forecasting System. Mon. Weather Rev. 136: 4773-4798.

Naud CM, DelGenio AD, Mace GG, Benson S, Clothiaux EE, Kollias P. 2008. Impact of dynamics and atmospheric state on cloud vertical overlap. J. Climate 21: 1758-1770.

Oreopoulos L, Barker HW. 1999. Accounting for subgrid-scale cloud variability in a multi-layer $1 \mathrm{D}$ solar radiative transfer algorithm. Q. J. R. Meteorol. Soc. 125: 301-330.

Oreopoulos L, Cahalan RF. 2005. Cloud inhomogeneity from MODIS, J. Climate 18: 5110-5124.

Oreopoulos L, Davies R. 1998. Plane-parallel albedo biases from satellite observations. Part II: Parametrisations for bias removal. J. Climate 11: $933-944$.

Pincus R, McFarlane SA, Klein SA. 1999. Albedo bias and the horizontal variability of clouds in sub-tropical marine boundary layers: Observations from ships and satellites. J. Geophys. Res. 125: 6183-6191.

Pincus R, Barker HW, Morcrette J. 2003. A fast, flexible, approximate technique for computing radiative transfer in inhomogeneous cloud fields. J. Geophys. Res. 108: DOI: 10.1029/2002JD003322.

Pincus R, Hannay C, Klein SA, Xu KM, Hemler RS. 2005. Overlap assumptions for assumed probability distribution function cloud schemes in large-scale models. J. Geophys. Res. 110: DOI: 10.1029/2004JD005100.

Pomroy HR, Illingworth AJ. 2000. Ice cloud inhomogeneity: Quantifying bias in emissivity from radar observations. Geophys. Res. Lett. 27: 2101-2104.

Räisänen P, Barker HW, Khairoutdinov MF, Li J, Randall DA. 2004. Stochastic generation of sub-gridscale cloudy columns for large-scale models. Q. J. R. Meteorol. Soc. 130: 2047-2067.

Räisänen P, Järvenoja S, Järvinen H, Giorgetta M, Roeckner E, Jylhä K, Ruosteenoja K. 2007. Tests of Monte-Carlo Independent Column Approximation in the ECHAM-5 atmospheric GCM. J. Climate 20: 4995-5011.

Randall DA, Coakley Jr JA, Fairall CW, Kropfli RA, Lenschow DH. 1984. Outlook for research on sub-tropical marine stratiform clouds. Bull. Amer. Meteorol. Soc. 65: 1290-1301.

Randall DA, Wood RA, Bony S, Colman R, Fichefet T, Fyfe J, Kattsov V, Pitman A, Shukla J, Srinivasan J, Stouffer RJ, Sumi A, Taylor KE. 2007. Climate models and their evaluation. Chapter 8 in Climate Change
2007: the Physical Science Basis. Contribution of Working Group I to the Fourth Assessment Report of the Intergovernmental Panel on Climate Change. Solomon S, Qin D, Manning M, Chen Z, Marquis M, Averyt KB, Tignor M, Miller HL. (eds.) Cambridge University Press: Cambridge, UK.

Rossow WB, Delo C, Cairns B. 2002. Implications of the observed mesoscale variations of clouds for the Earth's radiation budget. J. Climate 15: 557-585.

Shonk JKP, Hogan RJ. 2008. Tripleclouds: An efficient method for representing cloud inhomogeneity in $1 \mathrm{D}$ radiation schemes by using three regions at each height. J. Climate 21: 2352-2370.

Shonk JKP, Hogan RJ. 2010. Effect of improving representation of horizontal and vertical cloud structure on the Earth's global radiation budget. Part II: The global effects. Q. J. R. Meteorol. Soc. 136: 1205-1215, DOI: 10.1002/qj.646.

Slingo A. 1990. Sensitivity of the Earth's radiation budget to changes in low clouds. Nature 343: 49-51.

Smith SA, DelGenio AD. 2001. Analysis of aircraft, radiosonde and radar observations in cirrus clouds observed during FIRE II: The interactions between environmental structure, turbulence and cloud microphysical properties. J. Atmos. Sci. 58: 451-461.

Stephens GL, Wood NB, Gabriel PM. 2004. An assessment of the parametrisation of sub-gridscale cloud effects on radiative transfer. Part I: Vertical overlap. J. Atmos. Sci. 61: 715-732.

Tian L, Curry JA. 1989. Cloud overlap statistics. J. Geophys. Res. 94: 9925-9935.

Webb MJ, Senior CA, Sexton DMH, Ingram WJ, Williams KD, Ringer MA, McAvaney BJ, Colman R, Soden BJ, Gudgel R, Knutson T, Emori S, Ogura T, Tsushima Y, Andronova N, Li B, Musat I, Bony S, Taylor KE. 2006. On the contribution of local feedback mechanisms to the range of climate sensitivity in two GCM ensembles. Clim. Dyn. 27: $17-38$.

Wu X, Liang X. 2005. Radiative effects of cloud horizontal inhomogeneity and vertical overlap identified from a month-long cloud-resolving model simulation. J. Atmos. Sci. 62: 4105-4112.

Wu X, Moncrieff MW. 2001. Long-term behaviour of cloud systems in TOGA COARE and their interactions with radiative and surface processes. Part III: Effects on the energy budget and SST. J. Atmos. Sci. 58: $1155-1168$.

Yu W, Garand L, Dastoor AP. 1997. Evaluation of model clouds at $100 \mathrm{~km}$ scale using GOES data. Tellus A 49: 246-262. 